\title{
Incompatibilidade medicamentosa em terapia intensiva: revisão sobre as implicações para a prática de enfermagem
}

\author{
Graciele Oroski Paes ${ }^{1}$, Samara Oliveira Moreira ${ }^{2}$, Maiara Benevides Moreira ${ }^{3}$, Tamiris Gonçalves Martins ${ }^{4}$
}

\author{
${ }^{1}$ Enfermeira, Doutora em Enfermagem. \\ Professora Adjunta da Escola de \\ Enfermagem Anna Nery da Universidade \\ Federal do Rio de Janeiro. Rio de Janeiro, \\ RJ, Brasil. E-mail: \\ gracieleoroski@gmai.com. \\ ${ }^{2}$ Enfermeira, Mestre em Enfermagem. \\ Enfermeira do Instituto Estadual de \\ Cardiologia Aloysio de Castro. Rio de \\ Janeiro, RJ, Brasil. E-mail: \\ sassa.ufrj@bol.com.br. \\ ${ }^{3}$ Enfermeira. Discente do Programa de \\ Pós-Graduação em Enfermagem da \\ Universidade Federal do Rio de Janeiro. Rio \\ de Janeiro, RJ, Brasil. E-mail: \\ maiarabenevides@hotmail.com. \\ ${ }^{4}$ Enfermeira. Enfermeira do Hospital \\ Estadual Carlos Chagas e da UPA Bangu. \\ Rio de Janeiro, RJ, Brasil. E-mail: \\ tamiris ufrj@hotmail.com.
}

Recebido: 28/11/2015

Aceito: $12 / 04 / 2017$.

Publicado: 21/08/2017.

Como citar esse artigo:

Paes GO, Moreira SO, Moreira MB, Martins TG. Incompatibilidade medicamentosa em terapia intensiva: revisão sobre as implicações para a prática de enfermagem. Rev. Eletr. Enf. [Internet]. 2017 [acesso em: ____19:a20. Disponível em: http://dx.doi.org/10.5216/ree.v19.38718.

\section{RESUMO}

Revisão integrativa de literatura realizada em sete bases de dados, com objetivo de analisar a produção científica sobre as potenciais incompatibilidades medicamentosas em terapia intensiva e mapear os medicamentos incompatíveis de maior prevalência descritos na literatura. Os filtros aplicados foram: artigos disponíveis na íntegra, publicações do período de 2009 a 2016 nos idiomas português, inglês e/ou espanhol, totalizando 11 artigos ao término da seleção. A partir da revisão, observou-se que os medicamentos fenitoína e pantoprazol são os principais responsáveis pelas incompatibilidades medicamentosas nas unidades de terapia intensiva. 0 bloqueio das incompatibilidades pode ser realizado por meio de medidas simples implementadas rotineiramente pela enfermagem, tais como o aprazamento de medicamentos otimizado, a administração em vias venosas separadas de medicamentos sabidamente incompatíveis, a padronização da forma de diluição e administração de medicamentos, focando, principalmente, nos medicamentos incompatíveis de maior prevalência encontrados no estudo.

Descritores: Cuidados de Enfermagem; Cuidados Críticos; Incompatibilidade de Medicamentos; Cateteres Venosos Centrais; Administração Intravenosa.

\section{INTRODUÇÃO}

A incompatibilidade medicamentosa advém da diluição e/ou administração simultânea de dois medicamentos, ou mais, que interferem na eficácia terapêutica dos medicamentos e segurança do paciente, evidenciada visualmente por mudança de coloração da solução, precipitação ou turvação. Esse mecanismo se processa in vitro, ou seja, fora do organismo do paciente, o que o diferencia das interações 
medicamentosas propriamente ditas, já que estas ocorrem dentro do organismo ${ }^{(1)}$.

Além da presença de incompatibilidades entre dois medicamentos ou mais, este evento pode ser observado entre medicamentos e diluentes, entre medicamentos e adjuvantes e/ou entre medicamentos e os materiais dos cateteres venosos ${ }^{(2)}$.

Tais eventos ocorrem comumente em centros de terapia intensiva devido a múltiplos fatores, dentre eles o grande número de medicamentos prescritos e administrados diariamente. A administração de medicamentos de forma contínua concomitante e de forma intermitente corrobora para a ocorrência de incompatibilidades medicamentosas, principalmente quando o cuidado referente à compatibilidade e aprazamento dos horários de administração não são considerados ${ }^{(1,3-4)}$.

Somado a isto, observa-se que pacientes críticos poderão apresentar dispositivos de infusão intravenosa com vias reduzidas, propiciando a administração de medicamentos de forma concomitante, o que torna o risco de incompatibilidade aumentado ${ }^{(1-2)}$. Os dispositivos de infusão em forma de " $\mathrm{Y}^{\text {" também }}$ concorrem para as incompatibilidades, uma vez que há o encontro dos medicamentos na via final de infusão(2).

As incompatibilidades medicamentosas se classificam em químicas e físicas. As químicas ocorrem quando há a degradação de mais de 10\% de alteração molecular, sendo a hidrólise a reação mais frequente. Este tipo de reação é correlacionado à temperatura e pH da solução medicamentosa. Em contrapartida, as incompatibilidades físicas são evidenciadas pelas mudanças na coloração e viscosidade; precipitação; turvação da solução ou liberação de gases. Estas reações podem ocorrer imediatamente ao preparo ou tardiamente. Estima-se que eventos como este estão presentes em 3\% a $25 \%$ dos tratamentos administrados ${ }^{(2)}$, sendo responsáveis por $60 \%$ dos problemas graves e eventos adversos ocorridos nos hospitais ${ }^{(5)}$.

No âmbito da terapia intensiva, a principal via de administração medicamentosa é a intravenosa ${ }^{(6)}$. Essa via oferece maior segurança e efeito imediato das medicações no organismo. Nesse contexto, o cateter venoso central (CVC) torna-se o dispositivo de predileção por sua inserção em veias profundas e por ser suturado à pele, dificultando o desposicionamento. Além de tal fato, o tempo de permanência do dispositivo é maior se comparado ao acesso vascular periférico, o que configura sua preferência nestes setores.

Dessa forma, este estudo pretendeu investigar as incompatibilidades medicamentosas intravenosas mais frequentes, a fim de gerar um compilamento de evidências que apontem as melhores recomendações para mitigar os riscos potenciais envoltos no processo de segurança medicamentosa.

Para tanto, objetivou-se analisar a produção científica sobre as potenciais incompatibilidades medicamentosas em terapia intensiva e mapear os medicamentos incompatíveis de maior prevalência descritos na literatura.

Estudos que versem sobre os desdobramentos e implicações da segurança medicamentosa na prática assistencial em saúde, podem contribuir para melhorar a qualidade da assistência de enfermagem, além de reduzir as lacunas entre os preceitos teóricos e o cotidiano clinico. 


\section{MÉTODOS}

Revisão integrativa com adoção da estratégia PIO, que representa um acrônimo para Paciente, Intervenção e "Outcomes" (desfecho). Esses quatro elementos são fundamentais para a formulação da questão de pesquisa e para a busca bibliográfica de evidências, o que possibilita a definição de informações necessárias para a resolução da questão clínica de pesquisa ${ }^{(7)}$.

A revisão integrativa foi realizada abarcando as seguintes etapas: estabelecimento de hipóteses ou questões norteadoras, amostragem ou busca na literatura, categorização do estudo, avaliação dos estudos incluídos na revisão, interpretação dos resultados, síntese do conhecimento ou apresentação da revisão ${ }^{(8)}$.

A questão norteadora elaborada a partir da PIO centrou-se em: Quais as incompatibilidades medicamentosas intravenosas mais frequentes descritas na literatura no contexto da terapia intensiva?

Posteriormente realizou-se o levantamento de artigos com os seguintes Descritores em Ciências da Saúde (Decs): enfermagem, cuidados críticos, cateteres venosos centrais, administração intravenosa e incompatibilidade de medicamentos e do Medical Subject Headings (Mesh): nursing, critical care, central venous catheters, intravenous administration e drug incompatibility.

A seleção dos artigos foi realizada por dois doutores em enfermagem e dois enfermeiros assistenciais, apoiados na pergunta norteadora, selecionados conforme instrumento qualificador de produções científicas produzido pelos autores do estudo.

Assim, fez-se um levantamento de publicações científicas disponibilizadas nas bases de dados LILACS, SciELO, BDenf, CINAHL, MedLine, PubMed e SCOPUS. Foram utilizados os filtros: artigos disponíveis na íntegra, publicações do período de 2009 a 2016 nos idiomas português, inglês e/ou espanhol. Os critérios de inclusão foram: estudos observacionais, descritivos, analíticos; que trabalhem com seres humanos no âmbito da prática clínica, nos quais os autores sejam profissionais de saúde (médicos, farmacêuticos e enfermeiros). Como critério de exclusão adotou-se: artigos que não tratem de pacientes internados em terapia intensiva e duplicados. Foram pesquisados 1.927 artigos sendo selecionados para compor a amostra da pesquisa 11 artigos. 


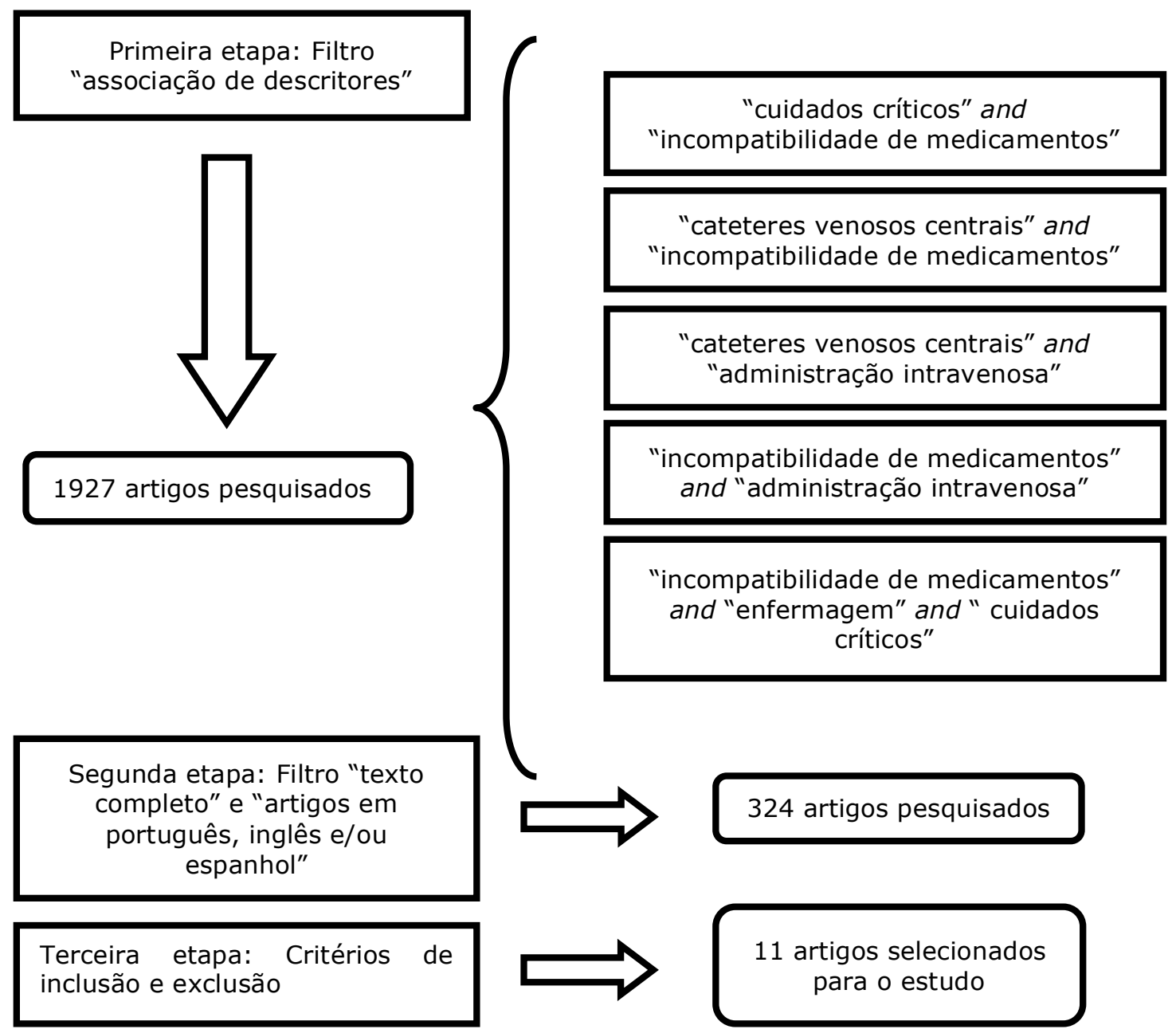

Figura 1: Fluxo de seleção dos artigos para compor a pesquisa.

\section{RESULTADOS}

Dos 1.927 artigos pesquisados, somente 11 atenderam aos critérios de seleção estabelecidos por esta revisão, sendo agrupados em duas categorias: fatores predisponentes para a ocorrência de incompatibilidade medicamentosa e incompatibilidades medicamentosas em terapia intensiva.

A fim de colaborar para otimização na leitura dos artigos selecionados, foi elaborado um quadro com os principais tópicos referentes a cada artigo (Quadro 1).

As produções selecionadas para compor a amostra desta pesquisa concentram-se em estudos produzidos e publicados em periódicos internacionais voltados para a área de farmácia. Como evidenciado na metodologia do estudo, as pesquisas encontradas e selecionadas desdobram-se em estudos originais realizados em unidades de terapia intensiva.

Com o caminhar da pesquisa, observou-se que duas principais categorias emergiram no tratamento dos dados, as quais são exemplificadas nos tópicos posteriores. 
Quadro 1: Síntese dos artigos incluídos na revisão.

\begin{tabular}{|c|c|c|c|}
\hline $\begin{array}{c}\text { Autores / Título / Ano de publicação / } \\
\text { Base de dados / País de origem }\end{array}$ & Delineamento & Intervenção & Resultados \\
\hline $\begin{array}{l}\text { Correard F, Savry A, Gauthier-Villano L, } \\
\text { Pisano P, Pourroy B / Visual } \\
\text { compatibility of defibrotide with } \\
\text { selected drugs during simulated Y-site } \\
\text { administration / } 2014 \text { / CINAHL / } \\
\text { Estados Unidos da América. }\end{array}$ & $\begin{array}{l}\text { Estudou-se o grau de compatibilidade visual de } \\
\text { uma solução de defibrotida com alguns tipos de } \\
\text { soluções de } 43 \text { medicamentos em concentrações } \\
\text { normalmente utilizadas na prática clínica. }\end{array}$ & $\begin{array}{l}\text { Foram analisados: anti-infecciosos, } \\
\text { corticoides, sedativos, analgésicos e agentes } \\
\text { cardiovasculares. As misturas foram } \\
\text { observadas imediatamente após e } \\
60 / 150 / 240 \text { minutos. Compatibilidade foi } \\
\text { definida como a ausência de mudança de cor, } \\
\text { turvação, partículas, geração de gás e } \\
\text { precipitação. }\end{array}$ & $\begin{array}{l}\text { Das } 43 \text { soluções testadas, } 36 \text { revelaram-se } \\
\text { visualmente compatíveis com a solução de } \\
\text { defibrotida ao longo de todo o período. Amicacina, } \\
\text { furosemida, midazolam, micofenolato de mofetila, } \\
\text { nicardipina, tobramicina e vancomicina são } \\
\text { incompatíveis com solução defibrotide. }\end{array}$ \\
\hline $\begin{array}{c}\text { Kumar A, Mann HJ / Visual } \\
\text { compatibility of oritavancin } \\
\text { diphosphate with selected } \\
\text { coadministered drugs during simulated } \\
\text { Y site administration / } 2010 \text { / CINAHL / } \\
\text { Estados Unidos da América. }\end{array}$ & $\begin{array}{l}\text { Estudou-se a compatibilidade visual do } \\
\text { oritavancin com vários medicamentos } \\
\text { comumente administrados a pacientes em } \\
\text { ambientes de cuidados intensivos. }\end{array}$ & $\begin{array}{l}\text { As soluções foram observadas por um período } \\
\text { de } 4 \text { horas em temperatura ambiente. } \\
\text { Compatibilidade foi definida como a ausência } \\
\text { de qualquer alteração de cor, fibras, } \\
\text { partículas e precipitado. }\end{array}$ & $\begin{array}{l}\text { Dos } 37 \text { fármacos testados, } 23 \text { eram visualmente } \\
\text { compatíveis com as três concentrações de } \\
\text { oritavancina durante o período de estudo de } \\
\text { quatro horas. Os fármacos formulados a um pH } \\
\text { básico ou neutro eram mais susceptíveis de serem } \\
\text { incompatíveis com a oritavancina. }\end{array}$ \\
\hline $\begin{array}{l}\text { Vijayakumar A, Sharon EV, Teena J, } \\
\text { Nobil S, Nazeer I / A clinical study on } \\
\text { drug related problems associated with } \\
\text { intravenous drug administration / } 2014 \\
\text { / PubMed / India. }\end{array}$ & $\begin{array}{l}\text { Estudo observacional prospectivo, realizado por } \\
\text { um período de } 4 \text { meses. Os pacientes que } \\
\text { recebem mais de dois medicamentos por via } \\
\text { intravenosa foram incluídos na pesquisa. }\end{array}$ & $\begin{array}{l}\text { Os objetivos foram avaliar os problemas } \\
\text { relacionados com a administração de } \\
\text { medicamentos intravenosos e desenvolver } \\
\text { estratégias para reduzir e prevenir a } \\
\text { ocorrência de erros durante a administração. }\end{array}$ & $\begin{array}{c}\text { Dos } 110 \text { pacientes estudados, } 80 \text { ( } 72,72 \%) \\
\text { relataram problemas relacionados a } \\
\text { medicamentos, sendo que } 61 \text { (55,4\%) problemas } \\
\text { foram observados em pacientes que receberam } \\
\text { medicamentos intravenosos através da linha } \\
\text { periférica. Destacam-se as incompatibilidades } \\
(40,9 \%) \text { como o problema mais evidente, seguido } \\
\text { dos erros na administração }(10,9 \%) \text { e erros de } \\
\text { diluição }(8 \%) \text {. }\end{array}$ \\
\hline $\begin{array}{l}\text { Westbrook JI1, Rob MI, Woods A, Parry } \\
\text { D / Errors in the administration of } \\
\text { intravenous medications in hospital } \\
\text { and the role of correct procedures and } \\
\text { nurse experience / } 2011 \text { / MedLine / } \\
\text { Inglaterra. }\end{array}$ & $\begin{array}{l}\text { Estudo observacional prospectivo que analisa o } \\
\text { preparo e administração de } 568 \text { medicamentos } \\
\text { intravenosos por } 107 \text { enfermeiros, em dois } \\
\text { hospitais de ensino. }\end{array}$ & $\begin{array}{l}\text { Os objetivos foram medir a frequência, tipo e } \\
\text { gravidade dos erros de administração } \\
\text { intravenosa em hospitais e as associações } \\
\text { entre falhas processuais e erros intravenosos } \\
\text { clínicos. Os erros foram identificados e } \\
\text { classificados por severidade. }\end{array}$ & $\begin{array}{l}\text { De } 568 \text { administrações intravenosas, } 69,7 \% \\
\text { apresentaram pelo menos um erro, sendo } 25,5 \% \\
\text { graves. Quatro tipos de erro (taxa intravenosa } \\
\text { errada, mistura, volume, e incompatibilidade de } \\
\text { medicamentos) foram responsáveis por } 91,7 \% \text {. As } \\
\text { taxas de erro e gravidade diminuíram com a } \\
\text { experiência clínica. Cada ano de experiência do } \\
\text { profissional reduziu o risco em 10,9\%. A } \\
\text { administração em bolus foi associada com um } \\
\text { aumento do risco de erro em } \mathbf{3 1 2} \% \text {. }\end{array}$ \\
\hline
\end{tabular}




\begin{tabular}{|c|c|c|c|}
\hline $\begin{array}{l}\text { Autores / Título / Ano de publicação / } \\
\text { Base de dados / País de origem }\end{array}$ & Delineamento & Intervenção & Resultados \\
\hline $\begin{array}{l}\text { Bertsche T, Münk L, Mayer Y, Stahl R, } \\
\text { Hoppe-Tichy T, Encke J, Haefeli WE / } \\
\text { One-year follow-up on procedure to } \\
\text { prevent i.v drug incompatibilities in an } \\
\text { intensive care unit / } 2009 \text { / MedLine / } \\
\text { Estados Unidos da América. }\end{array}$ & $\begin{array}{l}\text { Estudo de validação de um procedimento } \\
\text { operacional padrão (POP), com vistas a diminuir } \\
\text { a incompatibilidade de medicamentos. }\end{array}$ & $\begin{array}{l}\text { Foi avaliada a administração medicamentosa } \\
\text { de } 53 \text { pacientes em cuidados intensivos } \\
\text { objetivando a mitigação de } \\
\text { incompatibilidades medicamentosas através } \\
\text { de um POP. }\end{array}$ & $\begin{array}{l}\text { Dos } 2.014 \text { pares de medicamentos infundidos } \\
\text { simultaneamente através de todos os lúmens de } \\
\text { cateteres disponíveis, } 24 \text { pares eram incompatíveis. } \\
\text { O efeito da implementação do POP indicou que a } \\
\text { instituição do mesmo acompanhado por uma } \\
\text { reunião regular da equipe multiprofissional } \\
\text { melhorou a qualidade da terapia infusional. Este } \\
\text { seria, segundo o estudo, um método prático e } \\
\text { adequado para evitar erros na administração de } \\
\text { medicamentos. }\end{array}$ \\
\hline $\begin{array}{l}\text { Bertsche T, Veith C, Stahl A, Hoppe- } \\
\text { Tichy T, Meyer FJ, Katus HA, Haefeli WE } \\
\text { / A purging procedure for pantoprazole } \\
\text { and 4-lumen catheters to prevent IV } \\
\text { drug incompatibilities / } 2010 \text { / MedLine } \\
\text { / Alemanha. }\end{array}$ & $\begin{array}{l}\text { Estudo de intervenção, prospectivo. Realizado } \\
\text { em uma unidade de terapia intensiva } \\
\text { cardiovascular, onde protocolos operacionais } \\
\text { padrão sobre compatibilidade medicamentosa } \\
\text { eram utilizados. }\end{array}$ & $\begin{array}{l}\text { O objetivo foi avaliar o número de pacientes } \\
\text { com incompatibilidades antes e depois da } \\
\text { implementação de medidas de melhoria para } \\
\text { evitar incompatibilidades, que consistem em: } \\
\text { instrução acerca do pantoprazol e a } \\
\text { recomendação para usar cateteres com } 4 \\
\text { lúmens em substitutição de } 3 \text { lúmens, } \\
\text { aumentando o número de linhas de infusão } \\
\text { disponíveis. }\end{array}$ & $\begin{array}{l}\text { Em um ambiente de cuidados intensivos com boa } \\
\text { aderência ao procedimento operacional padrão, a } \\
\text { taxa de incompatibilidade diminui em relação a } \\
\text { ambientes que não possuem aderência, ao passo } \\
\text { que o uso de cateteres de } 4 \text { lumens em } \\
\text { substituição aos de } 3 \text { lumens não obteve o } \\
\text { benefício esperado nos pares de drogas } \\
\text { incompatíveis. }\end{array}$ \\
\hline $\begin{array}{l}\text { Cabezas CL, Guerrero L, Molas G, Soy D } \\
\text { / Physicochemical compatibility of high } \\
\text { concentration drugs usually Y-site } \\
\text { administered in intensive care units / } \\
2014 \text { / SCOPUS / Inglaterra. }\end{array}$ & $\begin{array}{l}\text { Estudo experimental. Foram estudadas as } \\
\text { incompatibilidades medicamentosas, sendo } \\
\text { definidas pela presença de turvação, } \\
\text { precipitação ou mudança de cor. }\end{array}$ & $\begin{array}{l}\text { Estudo demonstrou a compatibilidade físico- } \\
\text { química em Y de: dopamina ou dobutamina- } \\
\text { metadona em glicose } 5 \% \text { e solução fisiológica; } \\
\text { dobutamina-midazolam em dextrose 5\%; a } \\
\text { metadona-midazolam em dextrose } 5 \% \text { e } \\
\text { solução fisiológica, e metadona-esomeprazol } \\
\text { em solução fisiológica. }\end{array}$ & $\begin{array}{l}\text { Os resultados demonstraram a incompatibilidade } \\
\text { de: dobutamina com furosemida em solução } \\
\text { fisiológica; e midazolam com esomeprazol em } \\
\text { solução fisiológica. }\end{array}$ \\
\hline $\begin{array}{l}\text { Foinard A, Décaudin B, Barthélémy C, } \\
\text { Debaene B, Odou P / The impact of } \\
\text { multilumen infusion devices on the } \\
\text { occurrence of known physical drug } \\
\text { incompatibility: a controlled in vitro } \\
\text { study / } 2013 \text { / SCOPUS / Estados } \\
\text { Unidos da América. }\end{array}$ & $\begin{array}{l}\text { Estudo experimental. Foram estudados três } \\
\text { medicamentos: furosemida, midazolan e solução } \\
\text { fisiológica em concentrações e velocidades de } \\
\text { infusão diferentes em diversos dispositivos } \\
\text { intravenosos. }\end{array}$ & $\begin{array}{c}\text { A incompatibilidade física foi avaliada por dois } \\
\text { testes: inspeção visual e teste de contagem de } \\
\text { partículas visíveis de acordo com a } \\
\text { Farmacopeia Europeia. }\end{array}$ & $\begin{array}{c}\text { As características do dispositivo de infusão } \\
\text { parecem ter um impacto sobre a compatibilidade } \\
\text { física das duas drogas. } \\
\text { Sob condições específicas, o dispositivo de acesso } \\
\text { infusão múltiplo-lúmen impediu a } \\
\text { incompatibilidade entre a furosemida e o } \\
\text { midazolam. }\end{array}$ \\
\hline
\end{tabular}




\begin{tabular}{|c|c|c|c|}
\hline $\begin{array}{l}\text { Autores / Título / Ano de publicação / } \\
\text { Base de dados / País de origem }\end{array}$ & Delineamento & Intervenção & Resultados \\
\hline $\begin{array}{l}\text { Delaloye VH, Gryllaki MB, Voirol P, } \\
\text { Gattlen L, Pannatier A / In vitro } \\
\text { compatibility of various cardioactive } \\
\text { drugs during simulated Y-site } \\
\text { administration / } 2013 \text { / SCOPUS / } \\
\text { Inglaterra. }\end{array}$ & $\begin{array}{c}\text { Estudo experimental. Os medicamentos foram } \\
\text { diluídos da maneira usual realizada em unidades } \\
\text { de terapia intensiva. }\end{array}$ & $\begin{array}{l}\text { A compatibilidade foi verificada por inspeção } \\
\text { visual e por meio de ensaios químicos e } \\
\text { administração em Y simulada in vitro. As } \\
\text { soluções foram consideradas compatíveis na } \\
\text { ausência de qualquer alteração visual e de } \\
\text { qualquer variação significativa no valor de pH } \\
\text { e concentração de fármaco em cada } \\
\text { momento do estudo. }\end{array}$ & $\begin{array}{l}\text { Quando combinadas, as aminas cardioativas } \\
\text { mantiveram-se estáveis ao longo de } 24 \text { horas, com } \\
\text { a condição de serem protegidas da luz. }\end{array}$ \\
\hline $\begin{array}{l}\text { Marsilio NR, Silva D, Bueno D / } \\
\text { Incompatibilidades medicamentosas } \\
\text { em centro de tratamento intensivo } \\
\text { adulto de um hospital universitário / } \\
2016 \text { / SCOPUS -SCIELO / Brasil. }\end{array}$ & $\begin{array}{l}\text { Estudo transversal, prospectivo, quantitativo, } \\
\text { realizado em julho a setembro de } 2015 \text {. As } \\
\text { prováveis incompatibilidades foram identificadas } \\
\text { a partir da análise das prescrições dos pacientes } \\
\text { disponíveis no sistema on-line do hospital. }\end{array}$ & $\begin{array}{l}\text { Realizou-se uma intervenção farmacêutica } \\
\text { utilizando as diretrizes de preparo e } \\
\text { administração de medicamentos } \\
\text { incompatíveis. A adesão a essas diretrizes foi } \\
\text { posteriormente avaliada pela equipe de } \\
\text { enfermagem. } \\
\text { Foram analisadas } 100 \text { prescrições. }\end{array}$ & $\begin{array}{l}\text { Foram encontradas } 271 \text { incompatibilidades } \\
\text { medicamentosas, com média de } 4 \\
\text { incompatibilidades por prescrição. As mais comuns } \\
\text { foram: midazolam-hidrocortisona ( } 8,9 \%) \text {, cefepime- } \\
\text { midazolam }(5,2 \%) \text { e hidrocortisona-vancomicina } \\
(5,2 \%) \text {. A maior parte ocorreu quando um } \\
\text { medicamento foi administrado de forma contínua } \\
\text { e outro de forma intermitente ( } 50 \%) \text {. }\end{array}$ \\
\hline $\begin{array}{l}\text { Fang BX, Li P, Shi XY, Chen FC, Wang LH } \\
\text { / Incompatibilities of lornoxicam with } 4 \\
\text { antiemetic medications in polyolefin } \\
\text { bags during simulated intravenous } \\
\text { administration / } 2016 \text { / PubMed / } \\
\text { Estados Unidos da América. }\end{array}$ & $\begin{array}{l}\text { Estudo experimental. Foi avaliada a } \\
\text { compatibilidade e estabilidade de soluções } \\
\text { contendo lornoxicam com os } 4 \text { agentes } \\
\text { antieméticos em combinação para a analgesia } \\
\text { controlada pelo paciente, usualmente realizada } \\
\text { em os-operatório. }\end{array}$ & $\begin{array}{l}\text { As misturas dos analgésicos e antieméticos } \\
\text { com o lornoxicam foram inspecionadas } \\
\text { visualmente quanto a precipitação, turvação e } \\
\text { descoloração em cada intervalo de } \\
\text { amostragem, configurando, se evidente, } \\
\text { incompatibilidade entre os medicamentos. }\end{array}$ & $\begin{array}{l}\text { Após o armazenamento de } 4 \text { a } 48 \mathrm{hrs,} \mathrm{observou-se} \mathrm{a} \\
\text { presença de um ligeiro precipitado em todas as } \\
\text { combinações. Os resultados indicam que as } \\
\text { combinações de lornoxicam com droperidol, } \\
\text { ondansetrona, granisetron ou tropisetron em } \\
\text { solução de perfusão durante administração } \\
\text { intravenosa simulada foram incompatíveis. }\end{array}$ \\
\hline
\end{tabular}




\section{Incompatibilidades medicamentosas em cenários de terapia intensiva}

Nesta categoria foram selecionadas as principais incompatibilidades medicamentosas descritas na literatura atual; todas descritas no Quadro 2, têm a natureza química ${ }^{(9)}$.

Quadro 2: Principais incompatibilidades medicamentosas descritas na literatura pesquisada ${ }^{(10-14)}$.

\begin{tabular}{|c|c|c|c|}
\hline $\begin{array}{c}\text { Medicamento } \\
\text { base }\end{array}$ & $\begin{array}{c}\text { Medicamento secundário para } \\
\text { incompatibilidade }\end{array}$ & $\begin{array}{c}\text { Medicamento } \\
\text { base }\end{array}$ & $\begin{array}{c}\text { Medicamento secundário para } \\
\text { incompatibilidade }\end{array}$ \\
\hline \multirow{18}{*}{ Fenitoína } & Amicacina & \multirow{18}{*}{ Pantoprazol } & Amicacina \\
\hline & Gluconato de cálcio & & Fenitoína \\
\hline & Aciclovir & & Gluconato de cálcio \\
\hline & Ciprofloxacino & & Ciprofloxacino \\
\hline & Clindamicina & & Clindamicina \\
\hline & Dexametasona & & Dexametasona \\
\hline & Furosemida & & Hidrocortisona \\
\hline & Hidrocortisona & & Manitol \\
\hline & Manitol & & Metoclopramida \\
\hline & Metoclopramida & & Vancomicina \\
\hline & Pantoprazol & & Furosemida \\
\hline & Bicarbonato de sódio & & Metadona \\
\hline & Vancomicina & & \multirow{6}{*}{-} \\
\hline & Cloreto de potássio & & \\
\hline & Ranitidina & & \\
\hline & Fentanil & & \\
\hline & Midazolam & & \\
\hline & Noradrenalina & & \\
\hline \multirow{5}{*}{ Azitromicina } & Ciprofloxacino & \multirow{5}{*}{ Ciprofloxacino } & Clindamicina \\
\hline & Clindamicina & & Dexametasona \\
\hline & Amicacina & & Furosemida \\
\hline & Furosemida & & Hidrocortisona \\
\hline & Cloreto de potássio & & Bicarbonato de sódio \\
\hline \multirow{7}{*}{ Aciclovir } & Ciprofloxacino & \multirow{7}{*}{ Furosemida } & Ciprofloxacino \\
\hline & Pantoprazol & & Azitromicina \\
\hline & Fenitoína & & Fenitoína \\
\hline & \multirow{4}{*}{-} & & Pantoprazol \\
\hline & & & Vancomicina \\
\hline & & & Dobutamina \\
\hline & & & Midazolan \\
\hline \multirow{8}{*}{ Midazolan } & Hidrocortisona & \multirow{8}{*}{ Hidrocortisona } & Midazolam \\
\hline & Cefepime & & Vancomicina \\
\hline & Omeprazol & & Cloreto de cálcio \\
\hline & Fenitoína & & Vitamina B1 \\
\hline & Defibrotida & & Sulfametoxazol-trimetopim \\
\hline & Dopamina & & \\
\hline & Dobutamina & & - \\
\hline & Esomeprazole & & \\
\hline \multirow{8}{*}{ Defibrotida } & Amicacina & \multirow{8}{*}{ Lornoxicam } & Ondansetrona \\
\hline & Furosemida & & Droperidol \\
\hline & Midazolam & & Granisetron \\
\hline & Micofenolato de mofetil & & Tropisetron \\
\hline & Nicardipina & & \multirow{4}{*}{-} \\
\hline & Tobramicina & & \\
\hline & Vancomicina & & \\
\hline & Furosemida & & \\
\hline
\end{tabular}


As incompatibilidades medicamentosas mais citadas na literatura se relacionam aos medicamentos fenitoína e pantoprazol. Tais medicamentos possuem indicação diferenciada nos centros de terapia intensiva.

A fenitoína, por ser um medicamento anticonvulsivante, possui indicação restrita aos pacientes que apresentam algum distúrbio neuropsíquico. Já o pantoprazol, por ser um medicamento inibidor da bomba de prótons ${ }^{(15)}$ e, consequentemente, por oferecer proteção gástrica é indicado a todos os pacientes internados nos centros de terapia intensiva como forma de proteção à ulcera péptica de estresse.

Fatores predisponentes para a ocorrência de incompatibilidade medicamentosa

No levantamento realizado nesta pesquisa, foram abarcados os fatores que potencializam o aparecimento de incompatibilidades medicamentosas nas terapias intensivas. Corroborando com a literatura, esta revisão proporcionou o agrupamento das causas mais comuns de incompatibilidade medicamentosa.

Um achado discorre sobre a administração medicamentosa em bolus comparada a administração contínua, salientando que há o aumento de risco de $312 \%$ para incompatibilidade medicamentosa na administração em bolus ${ }^{(16)}$. Outro fator importante que contribui para a ocorrência das incompatibilidades medicamentosas é o número reduzido de linhas venosas para a administração de múltiplos medicamentos $^{(17)}$.

Ainda, é citado que não há um raciocínio clínico acerca da melhor indicação do número de lúmens individualizada a cada paciente, de forma que em alguns casos há um número de medicamentos muito superior a capacidade de administração pelo cateter, em detrimento de outro em que há um número superior de vias venosas para a administração de medicações em número reduzido ${ }^{(10-11)}$. Outro estudo trabalha com a ocorrência de incompatibilidades medicamentosas devido ao material em que o cateter é confeccionado, interagindo com o medicamento desta forma e propiciando possíveis incompatibilidades ${ }^{(10)}$.

\section{DISCUSSÃO}

A incompatibilidade é caracterizada como um erro associado ao uso de medicamentos, um evento que oferece risco com possibilidade de causar dano ao paciente internado em terapia intensiva, pois compromete a eficácia da terapêutica, interferindo de forma negativa na segurança medicamentosa.

Em vista disso, observa-se a importância do tratamento desta temática frente à assistência de enfermagem em centros de terapia intensiva. As incompatibilidades medicamentosas são relacionadas diretamente com o preparo e administração de medicamentos, sendo na maioria das vezes procedimentos realizados por profissionais da enfermagem e, portanto, o conhecimento acerca da melhor forma de realizálo deve ser reiterado.

A categorização dos estudos foi necessária para melhor análise dos dados. A primeira categoria intitulada "incompatibilidades medicamentosas em cenários de terapia intensiva" evidenciou os principais 
medicamentos incompatíveis segundo as literaturas pesquisadas, com destaque para fenitoína e pantoprazol.

A fenitoína é um medicamento anticonvulsivante, indicada para o tratamento de epilepsia. Possui utilização precisa para pacientes em terapia intensiva, no que tange ao tratamento de crises convulsivas, porém deve ser administrada de maneira isolada, pois seu potencial para gerar incompatibilidade é alto. Evidenciou-se 18 medicamentos que, se administrados simultaneamente à fenitoína na mesma via venosa, gerará uma reação de incompatibilidade medicamentosa. Os medicamentos listados como incompatíveis com a fenitoína abarcam a classe dos antibióticos, diuréticos, anti-eméticos, protetores gástricos, sedativos e aminas vasoativas ${ }^{(12-13)}$.

Porém, este medicamento não possui indicação para uso rotineiro nas terapias intensivas, de modo diferente do pantoprazol. Os pacientes internados em UTI estão constantemente sob estresse orgânico, o que pode propiciar o aparecimento de úlceras gástricas de estresse. Desta forma, torna-se rotineira a administração de medicamentos que previnam tal patologia e, portanto, há a indicação para a administração diária de inibidores da bomba de prótons, tais como omeprazol e/ou pantoprazol ${ }^{(15)}$.

Observa-se empiricamente que o aprazamento de enfermagem para tais medicamentos, omeprazol e pantoprazol, acontece às seis horas, minimizando possíveis interações com a alimentação, denominada interação fármaco-nutriente.

O aprazamento deste medicamento para as seis horas pode comprometer a identificação de possíveis reações decorrentes de uma incompatibilidade em função da transferência de cuidados/passagem de plantão, ocorrer em torno deste horário. Tal situação, pode implicar a não observação de reações químicas derivadas das incompatibilidades pela equipe que administrou o medicamento e, a equipe do plantão posterior dificilmente associará as reações observadas com uma reação de incompatibilidade, agregando os sinais apresentados a achados clínicos.

Não obstante, observa-se também que o aprazamento de enfermagem em unidades de terapia intensiva tem seguido um padrão rotineiro, no qual um grande aglomerado de medicamentos é aprazado para o mesmo horário, prejudicando a segurança medicamentosa, uma vez que o risco para as incompatibilidades medicamentosas aumenta proporcionalmente ao número de medicamentos prescritos e administrados conjuntamente ${ }^{(1-2)}$.

Desta forma, torna-se um cuidado de enfermagem a observação para um aprazamento de enfermagem com vistas a minimizar tais eventos indesejados. Preconiza-se, atualmente, a fuga de sistemas padronizados de aprazamento, nos quais há a estipulação de horários fixos, de forma a otimizar o rotina da terapia intensiva. Nesse sentido, propicia-se o aparecimento das incompatibilidades pela administração concomitante de diversos medicamentos no mesmo horário ${ }^{(4)}$.

O pantoprazol apresentou-se, de igual forma, como um medicamento propenso ao processo de incompatibilidade, segundo a literatura pesquisada, sendo evidenciado em 12 incompatibilidades medicamentosas. Tal fato torna-se importante quando observamos que tais incompatibilidades diminuem a 
eficácia terapêutica de pacientes em estado crítico, que dependem primordialmente do efeito dos medicamentos para que seu quadro clínico seja revertido e restaurado. A diminuição no efeito do pantoprazol pode gerar úlcera gástrica perfurante, levando à hemorragia digestiva alta, diminuição de hematócrito e hemácias circulantes, dificultando o desmame do ventilador mecânico. Portanto, é de suma importância que o conhecimento acerca das incompatibilidades perfaça o dia a dia do enfermeiro assistencial.

Dentre os medicamentos incompatíveis com o pantoprazol, podemos elencar a classe dos diuréticos, antibióticos, corticóides, antieméticos e soluções eletrolíticas. Tais medicamentos são extremamente importantes nas unidades de terapia intensiva e precisam ter seu potencial terapêutico dentro dos parâmetros normais para que se tenha a eficácia desejada.

Como forma de mitigar as incompatibilidades encontradas, à enfermagem cabe o aprazamento dos horários dos medicamentos pautados nas peculiaridades dos mesmos, com foco nas reações de incompatibilidades medicamentosas. A elaboração e instituição de procedimentos operacionais padrão também demonstrou ser uma estratégia efetiva para a minimização das incompatibilidades encontradas ${ }^{(17)}$.

Na segunda categoria emergida no estudo, intitulada como "Fatores predisponentes para a ocorrência de incompatibilidade medicamentosa", demonstra-se a importância da melhor escolha do acesso venoso e dos saberes referentes à administração de medicamentos para o bloqueio das incompatibilidades medicamentosas. O paciente crítico, pelo risco de instabilidade hemodinâmica, pela gravidade clínica e pelo uso de medicamentos irritantes, possui fortemente indicação para a inserção de cateteres venosos centrais. Estes cateteres possuem indicação precisa no contexto da terapia intensiva, porém podem apresentar situações indesejadas advindas de sua utilização.

Estudo ${ }^{(11)}$ comprovou que os dispositivos de infusão de múltiplo lúmen influenciam de forma direta a incompatibilidade medicamentosa, bloqueando-as entre midazolan e furosemida ${ }^{(11)}$. Desta forma, é importante que uma avaliação acerca do estado de saúde de cada paciente internado nas unidades de terapia intensiva seja realizada, assim como a observação do número de medicamentos prescritos e suas particularidades, de forma a propiciar uma melhor escolha do dispositivo de infusão e da quantidade de lúmens, propiciando um aumento da segurança medicamentosa, uma vez que bloqueia-se o risco de incompatibilidades.

Simultaneamente à tal fato, é importante observar os medicamentos que são administrados aos pacientes de forma contínua para que não haja incompatibilidade entre os mesmos nas vias do acesso venoso profundo. Em situações em que o número de medicamentos prescritos é maior do que o número de vias de administração, pode-se observar a utilização de dispositivos em " $\mathrm{"} \mathrm{ou} \mathrm{three} \mathrm{way.} \mathrm{Estes} \mathrm{dispositivos}$ permitem a infusão de mais de um medicamento pela mesma via do acesso venoso central, porém estes medicamentos são infundidos concomitantemente. Há de se atentar para a compatibilidade entre os medicamentos que são infundidos simultaneamente, pois esta forma de infusão pode propiciar as incompatibilidades medicamentosas ${ }^{(2)}$. 
Como produto final de uma incompatibilidade medicamentosa, podemos observar a formação de precipitados no equipo de infusão, recipiente de diluição ou via do cateter venoso, mudança de coloração da solução, turvação e formação de gases. Ressalta-se que tais reações podem diminuir a eficácia terapêutica e inferir de forma negativa no tratamento ofertado aos pacientes da terapia intensiva.

\section{CONCLUSÃO}

A incompatibilidade medicamentosa é um evento que apesar da disseminação de conhecimento na área de farmacologia, ainda necessita de um estudo aprofundado conforme evidenciado nesta pesquisa. Dos artigos, em 11 selecionados não foram destacadas produções de enfermagem, centrando-se apenas na área de farmacovigilância.

As incompatibilidades medicamentosas com medicamentos utilizados rotineiramente em unidades de terapia intensiva, tais como protetores gástricos (pantoprazol), anticonvulsivantes (fenitoína) e antibióticos ocorrem mais comumente. Porém, há uma grande defasagem em relação aos estudos que buscam investigar como a enfermagem poderia minimizar tais riscos aos pacientes.

O bloqueio das incompatibilidades pode ser realizado através de medidas simples implementadas rotineiramente pela enfermagem, como o aprazamento pautado nas características físico-químicas dos medicamentos, distribuição medicamentosa adequada nas vias do cateter venoso central, além da seleção do acesso venoso central de predileção para cada medicamento e da padronização da diluição e forma de infusão medicamentosa.

Para isso, é indispensável que haja investimentos na área de treinamento dos profissionais de saúde, para que os mesmos sejam capazes de identificar os medicamentos que influenciam no processo de incompatibilidade medicamentosa e intervenham, com vistas a sua mitigação.

Espera-se que este estudo possa contribuir para o esclarecimento sobre a ocorrência das incompatibilidades medicamentosas e suas peculiaridades de forma a subsidiar a equipe de enfermagem na minimização de tal evento nas unidades de terapia intensiva.

\section{REFERÊNCIAS}

1. Moraes CG, Silva D, Bueno D. Análise de Incompatibilidades de Medicamentos Intravenosos no Centro de Tratamento Intensivo Adulto do Hospital de Clínicas de Porto Alegre. Clinical and Biomedical Research [Internet]. 2011 [acesso em: 17 ago. 2017];31(1):31-8. Disponível em: http://seer.ufrgs.br/hcpa/article/view/14733.

2. Secoli SR, Pérez-Esquirol E, Heras-Matellán MJ, Vendrell-Bosh L, Ballarín-Alins E. Incompatibilidades en la terapia intravenosa: ¿qué hacer para prevenirlas? Enfermería Clínica [Internet]. 2009 [acesso em: 17 ago. 2017];19(6):349-53. Disponível em: http://dx.doi.org/10.1016/j.enfcli.2009.07.003.

3. Nemec K, Kopelent-Frank H, Greif R. Standardization of infusion solutions to reduce the risk of incompatibility. Am J Health Syst Pharm [Internet]. 2008 [acesso em: 17 ago. 2017];65(17):1648-54. Disponível em:

http://dx.doi.org/10.2146/ajhp070471. 
4. Silva LD, Barbosa SF, Prado ML, Sasso GTMD. Erros com medicamentos no contexto hospitalar: uma revisão bibliográfica. Evidentia [Internet]. 2011 [acesso em: 17 ago. 2017];8(34). Disponível em: http://www.indexf.com/evidentia/n34/ev7357p.php.

5. Santos L, Martinbiancho J, Tadiotto AL, Kreutz LM. Perfil das interações medicamentosas solicitadas ao Centro de Informações sobre Medicamentos de hospital universitário. Clinical and Biomedical Research [Internet]. 2011 [acesso em: 17 ago. 2017];31(3):326-35. Disponível em: http://seer.ufrgs.br/hcpa/article/view/22183.

6. Almeida SM, Gama CS, Akamine N, Nelson CS. Prevalência e classificação de interações entre medicamentos dispensadospara pacientes em terapia intensiva. Einstein (Säo Paulo) [Internet]. 2007 [acesso em: 17 ago.

2017];5(4):347-51. Disponível em: http://apps.einstein.br/revista/arquivos/PDF/672-

EinsteinOnLineTraduzidaVol5\%284\%29MioloPág347351.pdf.

7. Vieira LB, Reis A, Lima RE, Faria LM, Cassiani SHB. Interações Medicamentosas Potenciais em Pacientes de Unidades de Terapia Intensiva. Rev Ciênc Farm Básica Apl [Internet]. 2012 [acesso em: 17 ago. 2017];33(3):401-8. Disponível em: http://serv-bib.fcfar.unesp.br/seer/index.php/Cien_Farm/article/view/2009/1260.

8. Mendes KDS, Silveira RCCP, Galvão CM. Revisão integrativa: método de pesquisa para a incorporação de evidências na saúde e na enfermagem. Texto Context - Enferm [Internet]. 2008 [acesso em: 17 ago. 2017]; 17(4):758-64.

Disponível em: http://dx.doi.org/10.1590/S0104-07072008000400018.

9. Vijayakumar A, Sharon EV, Teena J, Nobil S, Nazeer I. A clinical study on drug-related problems associated with intravenous drug administration. J Basic Clin Pharm [Internet]. 2014 [acesso em: 17 ago. 2017];5(2):49-53. Disponível em: https://dx.doi.org/10.4103/0976-0105.134984.

10. Fernández-Ruiz M, Carretero A, Díaz D, Fuentes C, González JI, García-Reyne A, et al. Hospital-wide survey of the adequacy in the number of vascular catheters and catheter lumens. J Hosp Med [Internet]. 2014 [acesso em: 17 ago. 2017];9(1):35-41. Disponível em: http://dx.doi.org/10.1002/jhm.2130.

11. Foinard A, Décaudin B, Barthélémy C, Debaene B, Odou P. The Impact of Multilumen Infusion Devices on the Occurrence of Known Physical Drug Incompatibility. Anesth Analg [Internet]. 2013 [acesso em: 17 ago.

2017];116(1):101-6. Disponível em: http://dx.doi.org/10.1213/ANE.0b013e31826f5e02.

12. Marsilio NR, Silva D, Bueno D. Drug incompatibilities in the adult intensive care unit of a university hospital. Rev Bras Ter Intensiva [Internet]. 2016 [acesso em: 17 ago. 2017];28(2):147-53. Disponível em:

http://dx.doi.org/10.5935/0103-507X.20160029.

13. Correard F, Savry A, Gauthier-Villano L, Pisano P, Pourroy B. Visual compatibility of defibrotide with selected drugs during simulated Y-site administration. Am J Health Syst Pharm [Internet]. 2014 [acesso em: 17 ago.

2017];71(15):1288-91. Disponível em: http://dx.doi.org/10.2146/ajhp130700.

14. Fang BX, Li P, Shi XY, Chen FC, Wang LH. Incompatibilities of lornoxicam with 4 antiemetic medications in polyolefin bags during simulated intravenous administration. Medicine (Baltimore) [Internet]. 2016 [acesso em: 17 ago. 2017];95(25):e3824. Disponível em: http://dx.doi.org/10.1097/MD.0000000000003824.

15. Chinzon D, Boaventura S, Moraes-Filho JPP, Eisig JN, Laudanna AA. Inibidor de bomba de protônica pantoprazol em dose plena no tratamento das doenças ácido-relacionadas. Estudo ambulatorial brasileiro. RBM rev. bras. med [Internet]. 2001 [acesso em: 17 ago. 2017];58(1/2):17-20. Disponível em:

http://www.moreirajr.com.br/revistas.asp?fase=r003\&id_materia=1265.

16. Westbrook JI, Rob MI, Woods A, Parry D. Errors in the administration of intravenous medications in hospital and the role of correct procedures and nurse experience. BMJ Qual Saf [Internet]. 2011 [acesso em: 17 ago.

2017];20(12):1027-34. Disponível em: http://dx.doi.org/10.1136/bmjqs-2011-000089.

17. Bertsche T, Veith C, Stahl A, Hoppe-Tichy T, Meyer FJ, Katus HA, et al. A purging procedure for pantoprazole and 4lumen catheters to prevent IV drug incompatibilities. Pharm World Sci [Internet]. 2010 [acesso em: 17 ago.

2017];32(5):663-9. Disponível em: http://dx.doi.org/10.1007/s11096-010-9422-9. 